\title{
Autism Spectrum Disorder: Effects on Child and Family Functioning
}

\author{
Antoinette Donaldson ${ }^{\mathrm{a}}$, Tara Hays ${ }^{\mathrm{b}}$, Patrick W. Romani ${ }^{\mathrm{b}, \mathrm{c}}$
}

Children diagnosed with Autism Spectrum Disorders (ASD) are faced with a multitude of developmental deficits and behavioral challenges. These developmental deficits can be characterized by significant qualitative impairments in brain functioning, social skills, and language. The behavioral challenges can be characterized by physical aggression and restricted patterns of repetitive behaviors, interests, and activities. Both the developmental deficits and behavioral challenges can disrupt the entire family system. Research suggests that parents of children with ASD experience significantly higher levels of psychological distress and poor quality of life (QoL). Therefore, it is imperative that parents of children with ASD develop ongoing coping strategies that are effective to deal with their child's lifelong disability and to maintain their quality of life. This discussion paper will review literature on developmental/behavioral challenges associated with ASD. An emphasis will be placed on how these deficits effect the child and family functioning. Areas of future research for the helping professions will be discussed too.

Keywords: Autism Spectrum Disorder, developmental deficits, behavioral challenges, parents, quality of life, coping strategies

Many years ago, autism was considered to be a rare childhood disorder most often associated with the absence of meaningful expressive language, severe intellectual disabilities, and a lack of social awareness (American Psychological Association, 2017). Today, within the United States and in many places around the world, autism spectrum disorder (ASD) is now recognized as a common neurodevelopmental disorder. Roughly 1 in every 59 children are diagnosed with ASD. Boys are three-to-four times more likely to be diagnosed than girls (American Psychiatric Association, 2017). ASD is most often diagnosed in childhood, with symptoms typically becoming apparent before the age of 3 years, and is a lifelong disorder with relatively high comorbidity rates. While the exact cause is still unknown, many scientists and researchers believe that ASD is influenced by a variety of genetic and environmental factors that act during critical periods to alter key developmental processes (Matelski \& Van de Water, 2017).

According to the Diagnostic and Statistical Manual of Mental Disorders 5 ${ }^{\text {th }}$ Edition (DSM-5) "heritability estimates for autism spectrum disorder ranged from $37 \%$ to higher than $90 \%$, based on twin concordance rates" (p. 57). Because ASD is such a multifaceted neurodevelopmental disorder, it is important to understand the diagnostic criteria associated with it. A child is likely to be diagnosed with ASD (299.0 (F84.0)) if they have "persistent deficits in social communication and social interaction across multiple contexts, as manifested by the following: deficits in social-emotional reciprocity, deficits in nonverbal communication behaviors used for social interactions or deficits in developing, maintaining and understanding relationships" (American Psychiatric Association, 2013, p. 51). ASD is also characterized by "restricted, repetitive patterns of behavior, interests, or activities as manifested by at least two of the following: stereotyped or repetitive motor movements, use of objects, or speech, insistence on sameness, inflexible adherence to routines or ritualized patterns of verbal or nonverbal behavior, highly restricted, fixed interests are abnormal in intensity, or focus or hyper-or-hypo reactivity to sensory input or unusual interest in sensory aspects of the environment" (American Psychiatric Association, 2013, p. 51). Lastly, ASD "symptoms are present in the early developmental period and if the symptoms cause clinically significant impairment in social, occupational, or other important areas of current functioning and these symptoms are not better explained by intellectual disability" (American Psychiatric Association, 2013, p. 51).

Becoming a parent to a neurotypical child can have a dramatic impact on many individuals, both in terms of the stress they are likely to experience and its subsequent impact on marital satisfaction and emotional well-being. However, becoming a parent to a child born with a disability, such as ASD, can place an enormous amount of stress and demands on parents. Compared with parents of neurotypical children, parents of children with ASD have been found to experience significantly higher levels of parenting stress, psychological distress, and poor quality of life levels (Dardas \& Ahmad, 2013). Therefore, it is essential for parents of children with ASD to develop effective coping responses to help them deal with their child's lifelong disability as well as maintain their quality of life.

There is a surprising paucity of research investigating coping responses for caregivers of individuals diagnosed with ASD (Roane, Fisher, \& Carr, 2013). Much research addressing the developmental and behavioral differences associated with ASD focus on child-specific interventions (Roane et al., 2013). For parents to adequately implement these sometimes-timeconsuming interventions, it is imperative that they are psychologically healthy. Thus, the purpose of this literature review is to first describe the developmental and behavioral differences associated with ASD that have been shown to produce stress in caregivers. The issues of psychological distress, excessive stress levels, and low quality of life levels among parents of children with ASD will be described later. The issue of developing effective coping responses, interventions, and techniques to reduce stress and increase parent's quality of life will next be examined. The final sections in this paper will detail the gaps in the literature and implications for future research.

\section{Developmental and Behavioral Differences Associated with ASD}

a. Department of Psychology, Adams State University, 208 Edgemont Blvd, Alamosa, CO, 81101

b. Neuropsychiatric Special Care Unit, Children's Hospital Colorado

c. University of Colorado, Anschutz Medical Campus

Correspondence: antoinette.donaldson@childrenscolorado.org 
This first section of the current paper will highlight research describing the diagnostic characteristics associated with ASD. First, social deficits associated with ASD from a biologic and descriptive perspective will be described. Second, we will focus on language deficits associated, and third, behavioral excesses and deficits associated with ASD will be discussed.

Social Deficits. Per Laugeson, Frankel, Gantman, Dillon, and Mogil (2012), the most profound issues for individuals diagnosed with ASD are difficulties related to poor social functioning. Social deficits among individuals with ASD include perseverating on specific topics of interest; poor speech prosody (i.e., the rise and fall of voice pitch that happens naturally during speech); inability to carry out a two-way conversation and take turns speaking; being overly talkative in conversations with peers; and difficulty understanding and using humor, sarcasm, analogies, and metaphors (Laugeson et al., 2012). Deficits in the development of social skills often lead to substantial impairments in daily living, peer rejection, poor social support, and isolation. Thus, individuals diagnosed with ASD typically report feeling lonely, isolated and having poor quality of friendships (Laugeson et al., 2012).

A study conducted by Hazlett and colleagues (2017) was completed to identify differences associated with brain development in children diagnosed with ASD. They related these differences to subsequent social responses to common stimuli. Hazlett and colleagues conducted MRI's with 106 infants who were at high familial risk of ASD and 42 infants who were at a low familial risk for ASD. Neuroimaging data showed that children who had a high familial risk of ASD had bilateral enlargement of the amygdala and hippocampus in children with ASD compared to neurotypical children (Hazlett et al., 2017). The amygdala is known to play a crucial role in behavioral responses to stimuli and emotional learning. This would suggest that there is a relationship between the abnormal development of the amygdala and core symptoms of ASD, such as lack of shared enjoyment or poor reciprocal communication (Hazlett et al., 2017). Overall, these findings demonstrated that children diagnosed with ASD have brains that develop considerably larger in some areas compared to the brains of neurotypical children. These differences may affect their processing of common social stimuli (e.g., faces with different emotions). Understanding ASD related brain development is important for the further advancement of treatment and prevention as well as an overall understanding of the disorder.

Clearly, improving social skills among individuals with ASD is important. To address these deficits, Laugeson, and colleagues (2012) conducted a study to examine the effectiveness of the Program for the Education and Enrichment of Relational Skills (PEERS), a parent-assisted social skills group targeting social skills in children diagnosed with ASD. Thirty-three teens that had been previously diagnosed with ASD by the UCLA Autism Evaluation Clinic were selected and divided into two groups; a treatment group, which included individuals who received 14 weeks of treatment immediately after enrollment and a delayed treatment control group, which included individuals who waited 14 weeks for treatment after being enrolled. Both children with ASD and their parents attended separate counseling sessions that instructed them on key elements regarding social skills. The measures used in the study included: Kaufman Brief Intelligence Test - second edition, Vineland Adaptive Behavior Scales - second edition,
Social Skills Rating Scale (SSRS), The Quality of Play Questionnaire, Test of Adolescent Social Skills Knowledge, and Friendship Qualities Scale (Laugeson et al., 2012). A MANOVA was used to evaluate treatment results and it was revealed that parents in the treatment group reported overall greater improvement than parents in the delayed treatment control group (Laugeson et al., 2012). For example, the treatment groups significantly improved in their knowledge of social skills, yielding a value of $\mathrm{p}<0.01$, while the delayed treatment control group did not. The treatment group showed a significant increase in hosted get-togethers, yielding a value of $\mathrm{p}<0.01$, while the delayed treatment control group did not. There was no significance in either group regarding friendship quality. Overall, the findings indicated that the use of the PEERS program was effective in improving the social skills of individuals diagnosed with ASD and both parents and teachers could recognize a noticeable difference from members of both groups.

Language Deficits. Along with understanding brain development and social skills development in individuals diagnosed with ASD, it is important to also understand the development of language as well. According to Tager-Flusberg and Kasari (2013), roughly $30 \%$ of children diagnosed with ASD remain minimally verbal, even after receiving a wide range of educational interventions. Current literature addressing minimally verbal children with ASD is relatively sparse because most studies related to ASD diagnoses have focused on verbal individuals who are relatively higher functioning. This is because they can be evaluated more easily using standard assessment tools and are more compliant with experimental examinations, such as fMRI (Tager-Flusberg \& Kasari, 2013). Another reason the literature on minimally verbal children is sparse is that there is no clear definition of what being minimally verbal means. Individuals who lack all spoken language tend to use vocalizations and non-speech sounds as well as some vowel approximations. They may also utilize sign language, Picture Exchange Communication System (PECS) or Voice Output Communication Aids (VOCA) (Tager-Flusberg \& Kasari, 2013). Some individuals may exhibit limited expressive language through the use of a few words or fixed phrases, which are used infrequently and in limited situations (Tager-Flusberg \& Kasari, 2013). There are also individuals who have some spoken language, but that spoken language is predominantly echolalic or scripted. It is assumed that children who fail to acquire language have low nonverbal IQ scores. However, a recent study conducted by Munson et al. (2008) found that while some children that are minimally verbal have low nonverbal IQ scores, others do not. Likewise, while some minimally verbal children have low receptive and expressive language skills, other children who have minimal expressive language scores have good receptive language abilities (Rapin, Dunn, Allen, Stevens \& Fein, 2009). Gordon et al. (2010) conducted a study of a 12-year-old nonverbal boy diagnosed with ASD who knew just one single word. He began receiving highly intensive in home-based interventions with experienced clinicians, and throughout the next few years, he began to acquire a relatively large single word vocabulary (Gordon et al., 2010). The focus of the intervention was on speech-articulation skills but would change often to address specific individual needs. The significance of this case study is in demonstrating what might be possible during adolescent years (Gordon et al., 2010). 
Interventions for minimally verbal children with ASD include functional communication training, sign language, visual systems, PECS, and VOCA. Having very limited or no form of communication can cause a great deal of distress, not only for the child but also for the person with whom the child is trying to communicate with.

Behavioral Deficits (and Excesses). Research suggests that aggressive behaviors are common among children with ASD and are the primary cause for out of home, residential placement (Hill et al., 2014). The most common types of aggressive behaviors include hitting, kicking, biting, punching, spitting and scratching. Children with ASD also engage in selfinjurious behaviors such as head banging, which can have extremely detrimental effects. These aggressive behaviors are a source of parental concern and are known to increase stress within the family, financial strain and demands on caregivers (Hill et al., 2014). It has been found that aggressive behaviors may be influenced by the severity of ASD symptoms such as social and communicative deficits. Aggressive behaviors have also been linked to resistance to change and need for structure as well as increased repetitive, stereotyped and ritualistic behaviors (Hill et al., 2014). Increased aggressive behaviors are often associated with greater sleep difficulties, internalization of symptoms as well as hyperactivity and attention deficits (Hill et al., 2014). In order to better understand aggressive behaviors in children with ASD, Hill et al., (2014) conducted research which examined the prevalence of aggressive behaviors using the Child Behavior Checklist (CBCL), a wellvalidated parent questionnaire that measures emotional and behavioral problems. This study also looked at whether or not correlates such as age, gender, race, etc. that were associated with increased aggressive behavior in neurotypical children would also be associated with increased aggression in children with ASD. Lastly, Hill et al., (2014) wanted to examine the differences between children with and without aggressive behaviors in order to understand if children who do exhibit aggressive behaviors are diagnosed with more intensive medical conditions, demonstrate more severe impairments in behavioral functioning and experience more severe comorbid psychological symptoms. The study included 400 children between the ages of 2 and 18 with a confirmed ASD diagnosis who were enrolled in the Autism Speaks Autism Treatment Network at Oregon Health and Science University. The measures used for this study included sociodemographic information and the CBCL Aggressive Behavior scale. Medical treatments, behavioral functioning, and comorbidities were also taken into consideration when conducting this study (Hill et al., 2014). The results of this study indicated that the prevalence of aggressive behaviors in children with ASD is roughly $25 \%$ and the presence of aggressive behaviors is not associated with sociodemographic measures. It was found that children who do exhibit aggressive behaviors are more likely to be prescribed psychotropic medications and melatonin. There was also a significant link between aggressive behavior and overall cognitive functioning, sleep difficulties, and attention problems (Hill et al., 2014). Children with ASD may also exhibit restricted and repetitive behaviors that are not aggressive, but just as impactful on their lives and the lives around them. Restricted and repetitive behaviors include preoccupation with restricted interests, nonfunctional routines or rituals, repetitive motor mannerisms and persistent preoccupation with parts of an object. Understanding both aggressive and non-aggressive behaviors is crucial in understanding the life of a child with ASD.

\section{Effects of ASD on the Family System}

The challenges associated with raising a child with ASD are well known and well documented (Cameron, Dobson, \& Day, 1991; Koegel et al., 1992; Lecavalier, Leone, \& Wiltz, 2005; Sanders and Morgan, 1997; Smith et al., 2001; Wolf et al., 1989). Understanding the developmental deficits and behavioral challenges associated with ASD is a key component in understanding the high-stress levels, psychological distress, and low quality of life that parents so often report. According to Davis and Carter (2008), parents and clinicians agreed that problems in social skills, cognitive impairments, and aggressive behavior were the ASD-related symptoms that caused the most stress for parents.

Parent Stress. Parenting stress is defined as the experience of distress or discomfort resulting from the demands placed on a person as a result of their role as a parent (Deater-Deckard, 1998). Parents of children with ASD experience more parenting stress than parents raising typically developing children, children with Down syndrome, or children with behavior disorders (Bristol \& Schopler, 1984; Dumas, Wolf, Fisman, \& Culligan, 1991; Holroyd \& McArthur, 1976). Studies have shown that along with high levels of stress, parents of children with ASD also struggle with their own psychological distress, most often in the form of depression and anxiety (Davis \& Carter, 2008).

Studies of parent stress levels have indicated that the presence of a high level of ASD-related behaviors (e.g., repetitive motor movements) produces higher perceived parent stress levels (Wang et al., 2012; Phetrasuwan \& Shandor Miles, 2009; Konstantareas \& Homatidis, 2006). In a study of stress levels in parents of children and adolescents with ASD, Lecavalier and colleagues (2006) indicated that specific problem behaviors, for example externalizing behaviors, were more predictive of stress than adaptive behaviors. Davis and Carter (2008) reported higher levels of parenting stress in parents of toddlers with ASD. A longitudinal study was conducted in order to examine factors that are associated with high levels of parenting stress and psychological distress in parents of children with ASD. Thirty-nine percent of mothers reported parenting stress scores that were in the clinically significant range. Further analysis of the scores indicated that the most stressful areas in parenting children with ASD are related to the parent-child relationship. Depression and anxiety symptoms were also examined with $33 \%$ of mothers and $17 \%$ of fathers demonstrating depressive symptoms that fell in the clinically significant range. It was also reported that $6 \%$ of mothers and fathers alike had anxiety symptoms that were clinically significant. Overall, the results of this study indicated that there were high levels of parenting stress and psychological distress, specifically depressive symptoms, among parents raising a child with ASD.

Huang and colleagues (2014) examined the effects of specific autistic behaviors, emotional and behavioral problems on parent stress levels. The results indicated that parents of children with mild and moderate autistic behavior problems reported higher stress in the parent-child relationship in comparison to those parents of children with no or severe problems. Parents of children that displayed more prosocial behaviors indicated a lower stress level in the parent-child 
relationship. The highest parent stress levels were reported in relation to the child displaying conduct problems.

Dabrowsak and Pisula (2010) found higher stress levels in parents of children with ASD when compared to parents of typically developing children and parents of children with Down syndrome. The results indicated that mothers of children with ASD scored higher than fathers in parental stress. These same results were not indicated for parents of typically developing children or of children with Down syndrome.

Physical Stress. Parents of children with ASD not only experience decreased mental health, they also experience decreased physical health as well when compared to parents of neurotypical children (Johnson, Frenn, Feetham and Simpson, 2011). A Medical Outcomes Study (MOS) 36-item short-form health survey was given to 89 mothers and fathers of children with ASD. Results showed that 65 mothers and 24 fathers reported significantly lower physical health summary component scores compared to parents of neurotypical children (Johnson et al., 2011). In a study of 31 mothers and 30 fathers of children with ASD, it was found that mothers reported poorer physical health, as measured by the 12-item Short Form Health Survey (SF 12) than the control group of mothers of neurotypical children (Johnson et al., 2011). Johnson and colleagues (2011) found that the female's physical health was impacted the most by the physical demands of caring for a child with ASD, which becomes more physically demanding as the child ages.

Marital Stress. Marital distress is likely to occur when raising a child diagnosed with ASD because often times parents have different expectations for the future or plans on how to achieve certain milestones or goals, causing distress within the relationship (Johnson et al., 2011). Among 21 families of children with ASD, the majority of primary caregivers (66\%) reported that parenting stress effects family factors such as having minimal time for family outings and activities, lack of flexibility and spontaneity, as well as stress surrounding the marital relationship (Roa \& Beidel, 2009). According to Roa and Beidel (2009), parents of children with ASD also reported job-related stress, with fathers citing career restrictions and mothers reporting their inability to maintain employment or pursue activities outside of the home. In turn, this causes marital stress related to finances and job security. Furthermore, marital stress is attributed to the fact that many parents of children with ASD reported giving up on aspects of family life such as going on vacations or family outings (Roa \& Beidel, 2009).

Sibling Relationships. The literature examining the impact of children with ASD on siblings varies. Some studies revealed positive effects for siblings of children with ASD, such as increased positive self-concept when compared to matched controls (Roa \& Beidel, 2009). Other studies reveal that siblings of children with ASD have increased loneliness, depression and behavioral problems than siblings of neurotypical children (Roa \& Beidel, 2009). Roa and Beidel (2009) conducted a study, which examined sibling adjustment when living in a home with a sibling diagnosed with ASD. They did this by assessing siblings with the Piers-Harris Children's Self-Concept Scale, $2^{\text {nd }}$ edition, which provides a total self-concept score and six subscale scores. These six subscale scores included physical appearance, intellectual and school status, happiness and satisfaction, freedom from anxiety, behavioral adjustment, and popularity (Roa \& Beidel,
2009). While this study did not find a large number of differences between siblings of ASD and siblings of neurotypical children, one interesting finding was that siblings of ASD often internalize their problem behaviors more often (Roa \& Beidel, 2009). Overall, siblings of children with ASD have more behavioral problems than siblings of neurotypical children (Roa \& Beidel, 2009). However, when comparing brothers to sisters of siblings with ASD, it was noted that sisters had more positive self-concepts and sense of social competence than the brothers did (Roa \& Beidel, 2009).

Quality of Life. According to the World Health Organization (2018), quality of life is defined as "an individual's perception of their position in life in the context of culture and value systems in which they live and their relation to their goals, expectations, standards, and concerns". Yamada and colleagues (2012) conducted a study that examined the quality of life of parents caring for children with ASD. Of the 158 children (ranging in age from 6-15 years), they recruited 147 mothers and 122 fathers. The Medical Outcomes 36-item Short Form Health Survey (SF-36) was used to measure the quality of life of the parents. Parent's personality and marital relationships were assessed using the NEO Five Factor Inventory and the Intimate Bond Measure. The SF-36 results were compared to the national normative scores (Yamada et al., 2012). The results indicated that mothers had significantly low scores in the areas of social functioning, general health perceptions, vitality, emotions, and mental health. Overall, it was shown that mothers of children with ASD had lower quality of life scores compared to mothers of neurotypical children in the Japanese population (Yamada et al., 2012).

Support for Parents of Children with Autism. Given the stress associated with raising a child with ASD, it is not surprising that parents have elevated rates of depression and anxiety (Breslau \& Davis, 1986). Unfortunately, most of the intervention strategies available to assist parents are focused on parent training (Blackledge \& Hayes, 2006). Parent training is important for parents to understand and successfully manage their child's needs. However, a parent's psychological needs are often ignored throughout this process.

Some studies have shown families that include a child with ASD report lower levels of adaptability and cohesion in addition to lower levels of marital happiness when compared to norm groups (Higgins, Bailey, \& Pearch, 2005). It is also possible that raising a child with ASD can unite a family, creating a strong bond. A number of families report an increased resilience within the family as a result of raising a child with ASD (Bayat, 2007, p. 702). The family in fact becomes strong because of having a child with autism. Accessing psychotherapy is one way to build resiliency and address the multiple chronic and acute stresses of raising a child with ASD (Solomon \& Chung, 2012).

Coping Strategies. Stress experienced by parents of children with ASD may have a significant impact on their adjustment to taking care of their child (Dabrowska \& Pisula, 2010). Parents need to cope with stress to successfully adapt to parenting a child with ASD. The double ABCX model of family adaptation (McCubbin \& Patterson, 1983) considers coping the central process in a family's effort to adjust to a crisis. The double ABCX model defines family adaptations as a positive response to stressors using effective coping strategies. Much of the research on the stress of parents with children with developmental disabilities has focused on the 
specific challenges that parents face as opposed to the differences in coping strategies (Dabrowska and Pisula, 2010). Fleischman (1984) stated that individuals may have characteristic coping patterns of styles. Coping styles are dispositional variables which are stable characteristics in an individual. Studies indicate that emotion-oriented styles of coping (e.g. ruminating, blaming) is positively correlated with depression, anxiety, and somatic disorders (Endler \& Parker, 1990; Higgins \& Endler, 1995; Cohan et al., 2006). Taskoriented coping (e.g. active approach to problem solving) is negatively correlated with depression, anxiety, and somatic disorders.

Despite the challenges that parents of child with ASD experience there is evidence that parents can successfully develop coping skills (Bristol, 1984; Marcus et al., 1997).

Current literature shows multiple ways that caregivers can seek guidance and support in regards to reducing stress and increasing overall quality of life. Focusing on relaxation techniques such as deep breathing and positive self-talk creates an environment that enhances quality of life. Blackledge and Hayes (2006) conducted a 2-day (14 hour) workshop with a group of parents/guardians of children diagnosed with ASD. The workshop utilized Acceptance Commitment Therapy (ACT). ACT is a therapeutic approach that emphasizes the acceptance of unpleasant emotions and then focuses on the value driven goals. This unique approach to therapy is wellsuited for parents of children with autism. The focus is shifted from trying to find ways to make the inevitable challenges of raising a child with ASD go away to accepting the challenges moving forward in a way that honors their values and goals. The results of the study showed a reduction in psychological distress, psychiatric problems, and depression after the completion of the two-day workshop and at a three-month follow-up.

Respite Care. Parents of children with ASD report that a common source of stress is providing care for their child $24 \mathrm{~h}$ a day, 365 days a year (Doig et al., 2009). Respite care typically involves the temporary taking over of the parent role by an organized service on a regularly scheduled basis (Chan \& Sigafoos, 2001). According to Harper, Dyches, Harper, Roper and South (2013), respite care gives a family a short break and the opportunity "rest, refresh, relax, focus, regroup and recharge" (p. 5). Respite care allows for parents to shift the focus from the needs of the child with ASD to the needs of the parents or other family members. Respite care has been shown to reduce the stress that caregivers feel, have the ability to complete daily tasks that otherwise would not get done, allow parents to spend time with their other children and the ability to live a more ordinary life (Harper et al., 2013). Respite care, in the short-term, is correlated with significant reductions in parental stress, specifically for mothers (Chan \& Sigafoos, 2001). One study (Harper et al.) included 101 parents who had children with ASD; each of them was required to fill out a survey regarding marriage quality and daily hassle (2013). The results of this study concluded that respite care significantly increased marital quality and reduced overall familial stress (Harper et al., 2013).

Parent Training. For caregivers of children with ASD, education is a vital intervention in being able to reduce stress and improve overall quality of life. Raising a child with ASD involves multiple therapy sessions a week, which include but are not limited to, speech therapy, social skills training, behavioral training, etc. Having to keep up with the multitude of services these children need on a daily or weekly basis can be overwhelming. According to Binbin, Sun, Yi and Tang (2014), researchers examined the effects of parent education training in correlation to quality of life. Participants of this study were divided into two groups; one group was placed in a parent education-training program where they learned about their child's disorder as well as positive management strategies, while the other group was waitlisted and received no training. Results showed that through self-reported surveys, parents in the training program showed significantly increased results for quality of life. This included family functioning, self-efficacy, and positive coping styles.

\section{Gaps In Literature}

More research is needed in order to truly evaluate heightened stress levels, psychological distress and low quality of life in parents of children with ASD. When conducting research it was noted that many of the studies focused solely on maternal responsibilities and responses to stress, psychological distress, and quality of life. Many studies excluded the fathers or reported low participation by them. Another gap in literature pertains to the fact that many studies related to quality of life were not conducted within the United States; rather they were conducted in the Middle East, China or Japan. Cultural definitions of quality of life, depression, anxiety, and stress could be very different and therefore it is imperative to develop more literature related to those aspects within the United States.

\section{Implications for Counseling}

The immediate implication is the need for more awareness surrounding behavioral deficits and behavioral challenges associated with ASD. Additionally, clinical supervisors working with the ASD population need to develop competencies related to genetics and the environment as it is related to ASD. Furthermore, implementing early intervention strategies such as behavior therapy, speech therapy, and social skills therapy is imperative when dealing with a child diagnosed with ASD.

Further research could be conducted on how to counsel the child with ASD and the family as a whole. So much time is focused on the child that the parents are left with extreme burdens causing high stress levels and a low quality of life. Parents might be enrolled in counseling shortly after receiving a diagnosis of ASD for their child and given options for respite care. These early interventions for the parents and families of those afflicted by ASD have the potential to stave off the high stress levels and poor quality of life associated with raising a child with ASD. Further research conducted on the benefits of community support and how to obtain community support when raising a child with ASD should also be examined.

\section{Conclusion}

Autism spectrum disorders are becoming more prevalent throughout the world and each year more and more children are being diagnosed. Through the examination of research, it is evident that there are many developmental deficits and behavioral challenges that are associated with ASD. It is also apparent that because of these developmental deficits and behavioral challenges, parents of children with 
ASD are at an increased risk of high stress levels, psychological distress, and low quality of life. Deficits in social skills and language can create extreme barriers between children with ASD and their parents causing strained parentchild relationships. They can also create barriers between children with ASD and their peers, which results in isolation, poor social support, and low quality friendships. Behavioral challenges, such as physical aggression towards others and self-injury, also increases parental stress levels and decreases their quality of life. Being a parent to a physically aggressive and unpredictable child can cause extreme psychological distress and a very low quality of life.

However, hope is not lost. Studies have shown that implementation of coping strategies and interventions such as deep breathing and relaxation techniques, respite care and parent education has helped to reduce stress and improve quality of life. Children with ASD have so much to offer the world; their quirky ways, unique perspectives, and impeccable imagination can teach every single person the beauty of looking at the world through a different lens.

\section{References}

American Psychiatric Association. (2017). What Is Autism Spectrum Disorder? Retrieved July 11, 2018, from https://www.psychiatry.org/patientsfamilies/autism/what-is-autism-spectrum-disorder

Binbin, J., Sun, M., Yi, R., \& Tang, S. (2014). Multidisciplinary Parent Education for Caregivers of Children with Autism Spectrum Disorders. Archives of Psychiatric Nursing, 28319-326. doi:10.1016/j.apnu.2014.06.003

Dardas, L. A., \& Ahmad, M. M. (2013). Coping Strategies as Mediators and Moderators between Stress and Quality of Life among Parents of Children with Autistic Disorder. Stress and Health,31(1), 5-12. doi:10.1002/smi.2513

Davis, N., \& Carter, A. (2008). Parenting Stress in Mothers and Fathers of Toddlers with Autism Spectrum Disorders: Associations with Child Characteristics. Journal of Autism and Developmental Disorders,38, 1278-1291. doi:10.1007/s10803-007-0512-z

Dawson, G. (2008). Early behavioral intervention, brain plasticity, and the prevention of autism spectrum disorder. Development and Psychopathology,20(3), 775-803. doi:https://doi.org/10.1017/S0954579408000370

Gordon, K., Pasco, G., McElduff, F., Wade, A., Howlin, P., \& Charman, T. (2011). A communication-based intervention for nonverbal children with autism: What changes? Who benefits? APA PsycNET,79(4), 447-457. doi:10.1037/a0024379
Hazlett, H. C., Gu, H., Munsell, B. C., Kim, S. H., Styner, M., Wolff, J., . . Piven, J. (2017). Early brain development in infants at high risk for autism spectrum disorder. Nature - Techniques for Life Scientists and Chemists,542(7641), 348-351. doi:10.1038/nature21369

Hill, A. P., Zuckerman, K. E., Hagen, A. D., Kriz, D. J., Duvall, S. W., Van Santen, J., . . Fombonne, E. (2014). Aggressive Behavior Problems in Children with Autism Spectrum Disorders: Prevalence and Correlates in a Large Clinical Sample. National Institue of Health, 8(9), 1121-1133. doi:10.1016/j.rasd.2014.05.006

Harper, A., Dyches, T. T., Harper, J., Roper, S. O., \& South, M. (2013). Respite Care, Marital Quality, and Stress in Parents of Children with Autism Spectrum Disorders. Journal of Autism and Developmental Disorders,43(11), 2604-2616. doi:10.1007/s10803013-18120

Johnson, N., Frenn, M., Feetham, S., \& Simpson, P. (2011). Autism spectrum disorder: Parenting stress, family functioning and health-related quality of life. Families, Systems, \& Health,29(3), 232-252. doi:10.1037/a0025341

Laugeson, E. A., Frankel, F., Gantman, A., Dillon, A. R., \& Mogil, C. (2012). Evidence-Based Social Skills Training for Adolescents with Autism Spectrum Disorders: The UCLA PEERS Program. Journal of Autism and Developmental Disorders,42(6), 10251036. doi:10.1007/s10803-011-1339-1

Matelski, L., \& Van de Water, J. (2017). Risk factors in autism: Thinking outside the brain. Journal of Autoimmunity, 67, 1-7. doi:10.1016/j.jaut.2015.11.003

Rao, P. A., \& Beidel, D. C. (2009). The Impact of Children with High-Functioning Autism on Parental Stress, Sibling Adjustment, and Family Functioning. Behavior Modification,33(4), 437-451. doi:10.1177/0145445509336427

Rapin, I., Dunn, M., Allen, D., Stevens, M., \& Fein, D. (2009). Subtypes of language disorders in schoolage children with autism. Developmental Neuropsychology,34(1), 66-84. doi:https://doi.org/10.1080/87565640802564648

Tager-Flusberg, H., \& Kasari, C. (2013). Minimally Verbal School-Aged Children with Autism Spectrum Disorder: The Neglected End of the Spectrum. Autism Research,6(6), 1-15. doi:https://dx.doi.org/10.1002/aur.1329

Yamada, A., Kato, M., Suzuki, M., Watanabe, N., Akechi, T., \& Furukawa, T. A. (2012). Quality of life of parents raising children with pervasive developmental disorders. BMC Psychiatry, 12(119), 1-8. doi: doi:10.1186/1471-244X-12-119 